\title{
Deformations of Matrix Factorisations with Macaulay2
}

\author{
BRADFORD HOVINEN
}

ABSTRACT. We describe a Macaulay2 package which computes versal deformations of matrix factorisations, or equivalently maximal Cohen-Macaulay modules on hypersurfaces.

1. InTRODUCTION. We present here a Macaulay2 [M2] package, called Moduledeformations, which computes versal deformations of matrix factorisations of polynomials. We begin with some notation and definitions.

Definition 1.1. Let $S$ be a regular ring and $f \in S$ a regular element. A matrix factorisation of $f$ is a pair $(A, B)$ of square matrices of the same size over $S$ such that $A B=f \cdot I=B A$, where $I$ is the identity matrix of the same dimension.

Matrix factorisations of $f$ are equivalent to maximal Cohen-Macaulay (MCM) modules on the hypersurface defined by $f$ : given a matrix factorisation $(A, B)$ of $f$, the cokernel of $A$ is an MCM module over $S /(f)$, and all MCM modules over $S /(f)$ arise in this way; see [E].

For the course of this paper, we work with germs of varieties whose distinguished point is denoted by 0 . All morphisms $X \rightarrow Y$ map $0 \in X$ to $0 \in Y$. Our package represents a germ as the coordinate ring of an affine variety of which the germ is the localisation (or completion) at the ideal generated by the variables of the representing ring. It is an error if that ideal is not prime (e.g., if it is in fact the whole ring). With this in mind, we now define deformations of MCM modules.

Definition 1.2. Let $X$ be a germ of a hypersurface and $M_{0}$ a maximal Cohen-Macaulay module over $X$. Let $Y \rightarrow \Sigma$ be a flat map of germs whose fibre over $0 \in \Sigma$ is isomorphic to $X$. A deformation of $M_{0}$ in the deformation theory of $Y \rightarrow \Sigma$ is a pair $(S, \mathscr{M})$ with $S$ a germ of a variety equipped with a map $S \rightarrow \Sigma$ and $\mathscr{M}$ a module over $S \times{ }_{\Sigma} Y$, flat over $S$, whose restriction to $0{ }{ }_{\Sigma} X \subseteq S \times_{\Sigma} Y$ is isomorphic to $M_{0}$. In the sequel, we will supress from our notation the map $Y \rightarrow \Sigma$ when it is understood from context, referring simply to a deformation of $M_{0}$.

A deformation $\left(T, \mathscr{M}^{\prime}\right)$ is induced from $(S, \mathscr{M})$ if there exists a map $\phi: T \rightarrow S$ commuting with the maps $T \rightarrow \Sigma$ and $S \rightarrow \Sigma$ and such that $\mathscr{M}^{\prime} \cong\left(\phi \times_{\Sigma} \mathrm{id}_{Y}\right)^{*} \mathscr{M}$.

The deformation $(S, \mathscr{M})$ is versal if any deformation of $M_{0}$ is induced from $(S, \mathscr{M})$.

There are two cases which we discuss. In the absolute case, $\Sigma$ is a reduced point, and the module $M_{0}$ is deformed over $Y$ only. In the relative case, $\Sigma$ is the base space of a nontrivial deformation of $X$ and $Y$ is the total space of the deformation. In this case, $M_{0}$ is deformed also along the given deformation of $X$.

ModuleDeformations is an implementation of the Massey Product Algorithm for constructing versal deformations of maximal Cohen-Macaulay modules over hypersurfaces. The algorithm is described

2010 Mathematics Subject Classification. 14B12, $13 \mathrm{C} 14$.

ModuleDeformations version 1.0. 
in the absolute case in $[\mathrm{S}]$ and in general in $[\mathrm{H}]$. The implementation has been designed to take advantage of graded structure whenever possible. If the input is quasihomogeneous, then the output will be too, and the computation may run much more efficiently.

Below, we present three examples of the use of ModuleDeformations: one example in the absolute case, one simple example in the relative case, and one more complicated example in the relative case.

2. A Deformation in The ABSOlute CASE. The following Macaulay2 session illustrates the use of this package to construct a versal deformation of the maximal ideal $(x, y)$ of $\mathbb{Q}[[x, y]] /\left(x^{4}-y^{3}\right)$, which is maximal Cohen-Macaulay of rank one. First, we load the package and define the ring $\mathbb{Q}[x, y] /\left(x^{4}-y^{3}\right)$ in which we compute. This ring is graded if we set $\operatorname{deg} x=3$ and $\operatorname{deg} y=4$.

$$
\begin{aligned}
& \text { i1 : loadPackage "ModuleDeformations"; } \\
& \text { i2 : } \mathrm{R}=\mathrm{QQ}[\mathrm{x}, \mathrm{y} \text {, Degrees }=>\{3,4\}] /\left(\mathrm{x}^{\wedge} 4-\mathrm{y}^{\wedge} 3\right) \text {; }
\end{aligned}
$$

We now deform the module. The output is a pair $\left(\mathscr{O}_{S}, M\right)$, where $S$ is the base space of the deformation and $M$ is a module defined over the ambient space of $S \times Y$ whose restriction to $S \times Y$ is the deformation module. The procedure does not substitute the module $M$ to the ring of $S \times Y$ because the resulting computation of a Gröbner basis of the defining ideal of $\mathscr{O}_{S \times Y}$ is in certain cases too expensive.

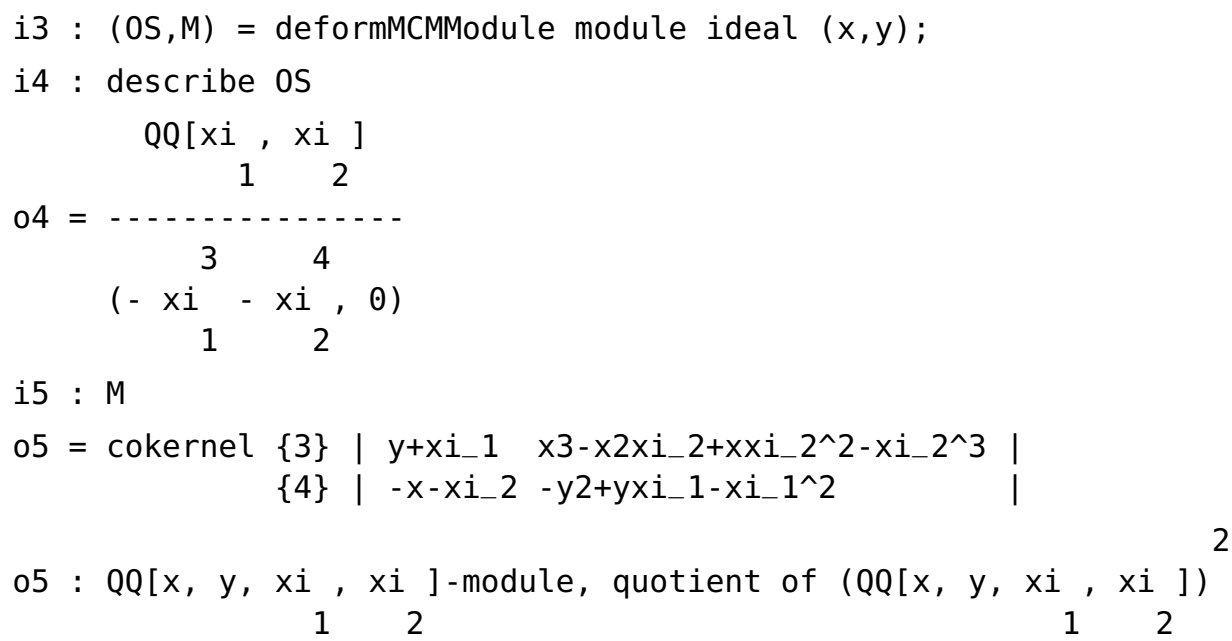

We may compare the presentation matrix of $M$ with that of the original module.

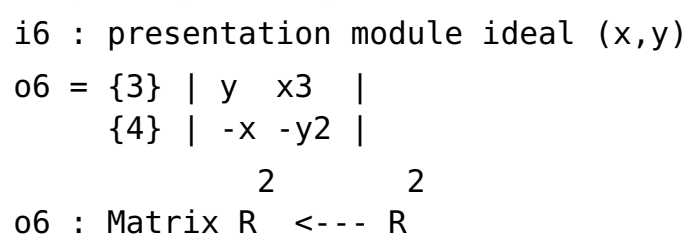

We see that the latter is obtained from the former by setting the deformation parameters $\xi_{1}$ and $\xi_{2}$ to 0 , as expected.

The maximal ideal has the same deformation theory as the residue field (see $[\mathrm{H}, \S 4.5]$ ). Thus the base space $S$ of its semiuniversal deformation is just the Hilbert scheme of one point, which is isomorphic to the original singularity $Y$. The substitution $\xi_{1} \mapsto y, \xi_{2} \mapsto x$ in the above output demonstrates this. The module is isomorphic to the defining ideal of the diagonal embedding of $Y$ into $Y \times S \cong Y \times Y$. 
3. A DEFormation in THE RElative CASE. In the following example we consider the Whitney umbrella $Y=\operatorname{Spec} \mathbb{Q}[[x, y, z]] /\left(z^{2}-(y-x) y^{2}\right)$. Reduction modulo $x$ gives the $A_{2}$ singularity $\operatorname{Spec} \mathbb{Q}[[y, z]] /\left(z^{2}-y^{3}\right)$. We deform the maximal ideal $(y, z)$ of the $A_{2}$ singularity in the deformation theory defined by the projection $\phi: Y \rightarrow \Sigma:=\operatorname{Spec} \mathbb{Q}[[x]]$.

First we define the rings $\mathscr{O}_{\Sigma}, \mathscr{O}_{Y}$ and the ring homomorphism $\phi^{*}: \mathscr{O}_{\Sigma} \rightarrow \mathscr{O}_{Y}$ corresponding to $\phi$. Setting $\operatorname{deg} x:=2, \operatorname{deg} y:=2$, and $\operatorname{deg} z:=3$ gives the associated affine rings a graded structure, which $\phi^{*}$ preserves.

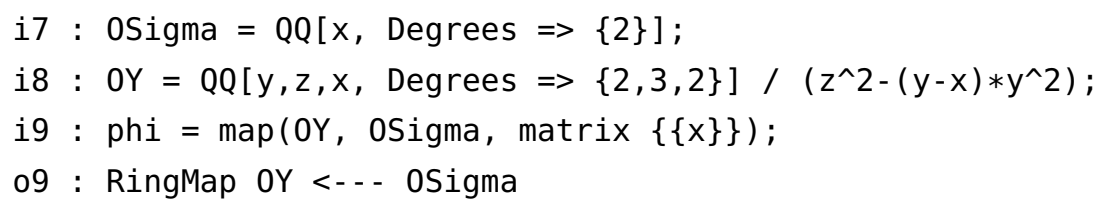

We then construct the ring $\mathscr{O}_{X}$ as the coordinate ring of the fibre over 0 of the map $Y \rightarrow \Sigma$. Over $\mathscr{O}_{X}$ we will construct the module $M_{0}$ to be deformed.

i10 : OX = trim (OY / phi ideal OSigma_O) ;

We now deform the maximal ideal $(y, z)$ of $\mathscr{O}_{X}$. The procedure returns the pair $\left(\mathscr{O}_{S}, M\right)$, where $\mathscr{O}_{S}$ is the ring of the base space and $M$ is a module defined over the ambient ring of $S \times_{\Sigma} Y$ whose restriction to $S \times{ }_{\Sigma} Y$ is the deformation module. The procedure does not substitute the module $M$ into the ring of $S \times{ }_{\Sigma} Y$ for the same reason as in the absolute case above.

ill : $(S, M)=$ deformMCMModule(module ideal $(y, z)$, phi);

i12 : describe $S$

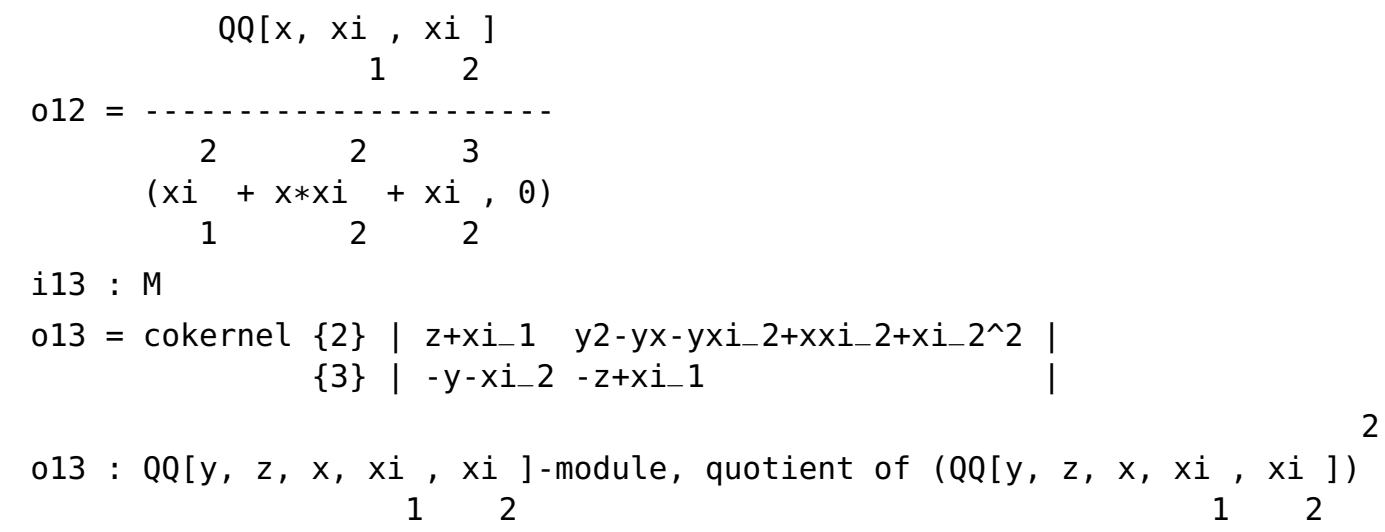

Similar to the example of $\S 2$, the base space $S$ of the deformation is just the Hilbert scheme of one point on the Whitney umbrella $Y$. The substitution $y \mapsto-\xi_{2}, z \mapsto \xi_{1}$ establishes this isomorphism in the output above. The module $M$ is again isomorphic to the defining ideal of the diagonal embedding of $Y$ in $Y \times_{\Sigma} S \cong Y \times_{\Sigma} Y$.

4. A MORE COMPLICATED EXAMPLE. In the following example, we compute a versal deformation of the normalisation of the $E_{6}$ curve singularity onto the discriminant in a semiuniversal deformation of the $A_{3}$ singularity. A semiuniversal deformation of the $A_{3}$ singularity $\operatorname{Spec} \mathbb{C}[[x, y]] /\left(x^{4}-y^{2}\right)$ is given by the equation $x^{4}-y^{2}+6 a_{2} x^{2}+4 a_{3} x+3 a_{4}=0$, with base space $\operatorname{Spec} \mathbb{C}\left[\left[a_{2}, a_{3}, a_{4}\right]\right]$. The assignment of degree $i$ to each parameter $a_{i}$, along with $\operatorname{deg} x=1$ and $\operatorname{deg} y=2$, makes the equation quasihomogeneous. The discriminant is a hypersurface in $\operatorname{Spec} \mathbb{C}\left[\left[a_{2}, a_{3}, a_{4}\right]\right]$ defined by the equation

$$
a_{4}^{3}-a_{3}^{4}+6 a_{2} a_{3}^{2} a_{4}-6 a_{2}^{2} a_{4}^{2}-2 a_{2}^{3} a_{3}^{2}+9 a_{2}^{4} a_{4}=0 .
$$


which is quasihomogeneous of degree 12 .

The $E_{6}$ singularity $a_{4}^{3}-a_{3}^{4}=0$ is obtained by intersection with the hyperplane $a_{2}=0$. The Macaulay 2 code below constructs the ring $R$ of the discriminant, the map $\operatorname{Spec} R \rightarrow \Sigma \cong \operatorname{Spec} \mathbb{C}\left[\left[a_{2}\right]\right]$, and the ring $\tilde{R} \cong \operatorname{Spec} \mathbb{C}\left[\left[a_{3}, a_{4}\right]\right] /\left(a_{4}^{3}-a_{3}^{4}\right)$ of the $E_{6}$ singularity.

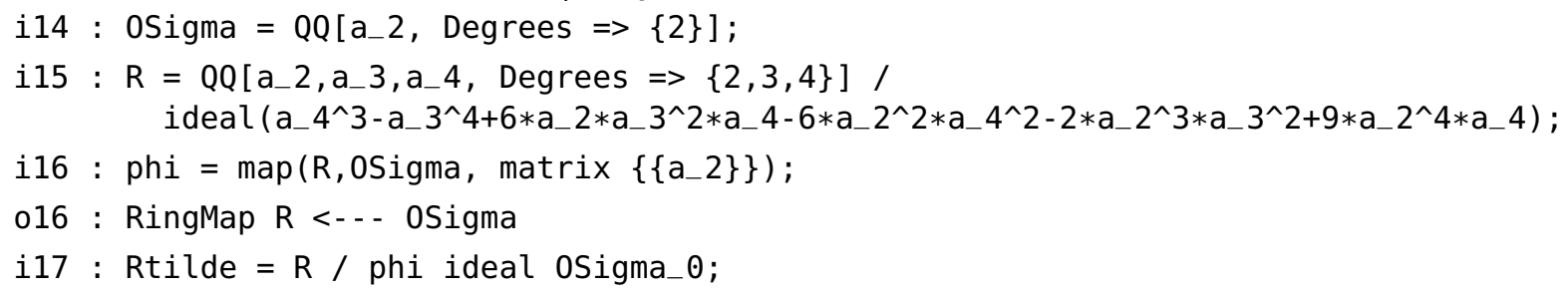

The normalisation $N(\tilde{R})$ of $\tilde{R}$, obtained by the parametrisation $\tilde{R} \rightarrow \mathbb{C}[[t]]$ given by $a_{3} \mapsto t^{3}, a_{4} \mapsto t^{4}$, has presentation matrix

$$
\left[\begin{array}{ccc}
a_{4} & 0 & -a_{3}^{2} \\
-a_{3} & a_{4} & 0 \\
0 & -a_{3} & a_{4}
\end{array}\right] .
$$

As modules over $\tilde{R}, N(\tilde{R})$ is isomorphic to the conductor ideal $\left(a_{3}^{2}, a_{3} a_{4}, a_{4}^{2}\right)=\left(a_{3}, a_{4}\right)^{2}$ of the normalisation map. Thus we construct and deform the module:

i18: $(S, M)=$ deformMCMModule $\left(\operatorname{module}\left(\text { ideal }\left(a_{-} 3, a_{-} 4\right)\right)^{\wedge} 2\right.$, phi $)$;

We may then investigate $S$ :

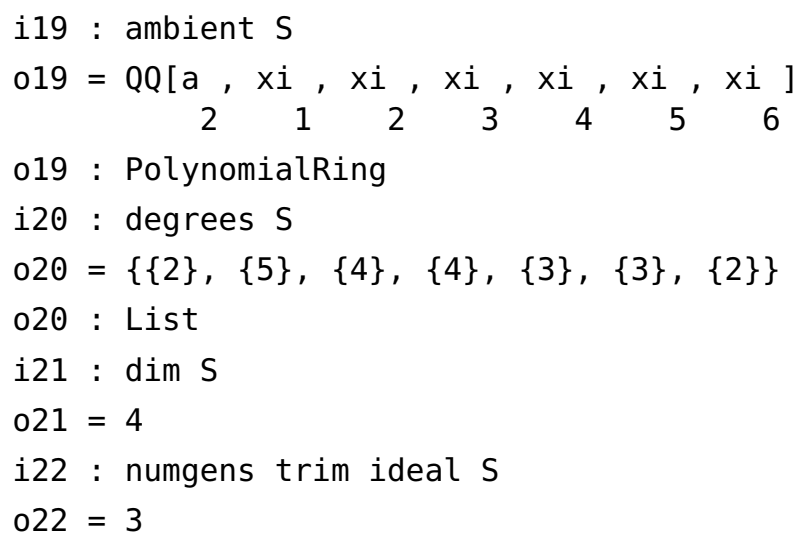

There are six parameters $\xi_{1}, \ldots, \xi_{6}$ with degrees $\operatorname{deg} \xi_{1}=5, \operatorname{deg} \xi_{2}=4=\operatorname{deg} \xi_{3}, \operatorname{deg} \xi_{4}=3=\operatorname{deg} \xi_{5}$, and $\operatorname{deg} \xi_{6}=2$. The base space is a complete intersection of dimension 4 in a seven-dimensional ambient space.

Setting $a_{2}$ to 0 in the above output recovers a versal deformation of the normalisation of $\tilde{R}$ in the absolute case. Such a deformation is among those constructed in [S], and indeed the results therein may be recovered using this Macaulay2 module. A direct comparison requires applying to the base space of the deformation a change of variables which can be done by (somewhat tedious) inspection.

\section{REFERENCES.}

[E] D. Eisenbud, Homological algebra on a complete intersection, with an application to group representations, Trans. Amer. Math. Soc. 260 (1980), no. 1, 35 - 64.

[M2] D.R. Grayson and M.E. Stillman, Macaulay 2, a software system for research in algebraic geometry, available at Ww. math . uiuc . edu/Macaulay2/.

[H] B. Hovinen, Matrix factorizations of the classical discriminant, $\mathrm{PhD}$ thesis, University of Toronto, 2008. 
[S] A. Siqveland, The method of computing formal moduli, J. Algebra 241 (2001), no. 1, 292 - 327.

RECEIVED : 2008-11-09 REVISED : 2009-12-11 ACCEPTED : 2010-04-17

hovinen@math. uni-hannover. de : Institut für Algebraische Geometrie, Leibniz Universität Hannover, Welfengarten 1, 30167 Hannover, Germany. 